\title{
Variations
}

Variations

Revue internationale de théorie critique

$9 / 10 \mid 2007$

\section{Les frontières de la politique}

\section{Pour une anthropolitique}

\section{Edgar Morin}

\section{(2) OpenEdition}

\section{Journals}

Édition électronique

URL : http://journals.openedition.org/variations/463

DOI : 10.4000/variations.463

ISSN : 1968-3960

\section{Éditeur}

Les amis de Variations

\section{Édition imprimée}

Date de publication : 1 juin 2007

Pagination : 9-27

\section{Référence électronique}

Edgar Morin, «Pour une anthropolitique », Variations [En ligne], 9/10 | 2007, mis en ligne le 01 décembre 2012, consulté le 01 mai 2019. URL : http://journals.openedition.org/variations/463 ; DOI : $10.4000 /$ variations.463

Ce document a été généré automatiquement le 1 mai 2019.

Les ami•e•s de Variations 


\title{
Pour une anthropolitique
}

\author{
Edgar Morin
}

\section{NOTE DE L'ÉDITEUR}

\author{
Entretien réalisé par Wilfried Graf et Alex Neumann
}

Vous avez l'ambition de proposer une compréhension théorique cohérente. Or, l'époque est plutôt à la méfiance postmoderne vis-à-vis des grands systèmes.

Une méthode cohérente n'est pas assimilable à un grand système. La cohérence, c'est la tentative de l'esprit pour affronter la complexité du réel. Mon problème, c'est donc d'affronter les contradictions qui se présentent à l'esprit, sans toujours leur chercher une solution. Mon propos sur la méthode ne s'inscrit pas prioritairement en réaction à ce qu'on appelle le postmodernisme - sur lequel, d'ailleurs, il y aurait beaucoup à dire. Il s'inscrit plutôt en réaction à un mode de connaissance morcelé, fragmenté, qui est délivré dans tous nos enseignements - par exemple à l'université. Celui-ci empêche de mettre en contexte les informations ou les faits dont il s'occupe. De plus, il empêche de situer dans un ensemble plus global, plus ample, ces informations ou ces données. Je réagis donc surtout contre une façon mutilante d'appréhender la réalité. Par ailleurs, le terme de « postmoderne » est très insuffisant pour caractériser notre époque. D'abord, parce que, conceptuellement, il est vide. Ensuite, je pense que notre époque n'a pas encore montré son vrai visage. Il est trop tôt pour que cela soit intéressant de la qualifier. Une des caractéristiques du postmoderne, tel que Lyotard l'a développé, c'est la fin des grands récits. Il est vrai que certains moyens récits, comme le récit marxiste, qui prétendait fournir une interprétation globale de l'histoire de l'humanité - avec le communisme primitif comme point de départ, puis le monde féodal, puis le capitalisme, puis enfin le socialisme - ne fonctionnent plus. En revanche, depuis 1960, nous disposons du plus grand récit qu'on puisse imaginer. Il débute, il y a 15 milliards d'années, avec la naissance du cosmos. Puis vient la formation, il y a 5 milliards d'années, de notre soleil, lui-même issu de la décomposition d'un soleil antérieur. Au milieu de tout cela, notre planète Terre s'est formée, où la vie est apparue. Enfin, dans 
le cours de cette évolution biologique, il y a un processus qui date d'il y a 6 millions d'années, qui s'appelle l'hominisation, et qui aboutit à notre espèce actuelle. Jamais, on n'avait imaginé un récit aussi gigantesque, depuis les origines de l'univers jusqu'à aujourd'hui, et qui va évidemment se poursuivre.

En ce qui concerne les formes de développement de ce que vous appelez la pensée complexe, vous évoquez la nécessité du passage de la dialectique à un concept biologique. Vous dites qu'on ne peut plus parler de synthèse au sens hégélien, et qu'il y a une coopération des contradictions en mouvement. Expliquez-nous un peu ce schéma.

Ce qu'on appelle dialectique - au sens d'une pensée qui affronte des antinomies ou des contradictions - est un type de pensée qui s'est développé de manière très marginale ou minoritaire dans le monde occidental. Héraclite représente un premier grand jalon. Il développe une pensée affrontant la contradiction sans chercher à la dépasser. Quand il dit: "Vivre de mort ou mourir de vie», cela ne signifie pas qu'il est possible de dépasser cette opposition. C'est une manière de dire que la vie, par exemple celle d'un individu, ne peut se poursuivre qu'à travers la mort de ses molécules et de ses cellules, et leur remplacement par des molécules et des cellules nouvelles. La vie tire profit du fait d'être soumise à la mort. De même, les sociétés vivent de la mort des individus. Cette contradiction ne supprime pas le caractère néfaste de la mort, qui est irrémédiable: on finit toujours par mourir. Elle indique seulement que la vie est capable d'utiliser la mort pour lutter contre la mort. C'est la définition de la vie que donne Bichat au XIXe siècle: «La vie est l'ensemble des fonctions qui résistent à la mort ». Il faut ajouter : en utilisant la mort elle-même. La dialectique, c'est donc une pensée qui, non seulement affronte les contradictions, mais voit ce qui les lie de façon inséparable. C'est l'affirmation de l'unité des contraires sans qu'ils cessent d'être contraires dans l'unité. Rien à voir avec l'idée d'une unité qui supprime les contraires. Hegel apporte l'idée que le choc des antagonismes, des contradictions, produit un dépassement (Aufhebung) assimilable à une synthèse nouvelle.

Naturellement, pour Hegel, cette synthèse nouvelle n'est pas un point final, c'est le point de départ d'un nouveau jeu ininterrompu. C'est l'une des raisons pour lesquelles je préfère parler de "dialogique " plutôt que de dialectique. Quand on utilise le mot dialectique, on pense trop souvent à Hegel et à ce que Marx lui emprunte : l'idée d'un dépassement des contradictions. Je ne dis pas que les contradictions sont toutes indépassables. Je dis qu'il y a des contradictions créatrices, c'est-à-dire à partir desquelles naît quelque chose de nouveau. Je me réfère ici un peu à Castoriadis, qui insiste sur le caractère créateur qu'il peut y avoir aussi bien dans l'évolution vivante, biologique, que dans l'histoire humaine. Je pense que les créations naissent de réponses à des défis, de situations impossibles que l'on affronte en créant quelque chose de nouveau, qui n'est pas la synthèse des termes en conflit.

La seconde raison pour laquelle je me distingue de Hegel, c'est que, dans sa logique dialectique, tout part de l'être qui, étant vide de toute détermination, équivaut au néant. De cette tension, entre l'un et l'autre, naît le devenir. Or, je considère que dans la réalité, il n'y a pas seulement le deux qui naît du un, il y a aussi le un qui naît du deux. Pas seulement dans le sens de la synthèse qui réunit des gens, mais dans le sens de la rencontre entre deux étrangers. Ce que je veux dire, c'est que le rôle de la rencontre et du hasard, qui était un concept ignoré à l'époque de Hegel, est extrêmement important. Et c'est aussi pour cela, finalement, que j'ai préféré le terme de dialogique. 
Le terme de dialogique suggère que l'équilibre est une notion provisoire, précaire. La priorité reviendrait au déséquilibre, à la lutte entre éléments contraires.

Pour tout ce qui est vivant, il vaut mieux parler, à mon avis, de régulation plutôt que d'équilibre. La régulation, c'est quand un système s'auto-entretient, entretient sa constance, sa régularité, par l'élimination de la déviance. Ce que l'on appelle l'homéostasie pour le corps humain, c'est toute une série de processus qui nous maintiennent à la même température, avec les mêmes taux de différents éléments chimiques dans notre sang, etc. Les grands phénomènes qu'on appelle d'équilibres sont, en fait, des phénomènes de régulation: "élimination de la déviance qui, si elle s'accroît, risque de détruire le système " - disons le feed-back positif. À la lumière de cela, on peut reconsidérer un certain nombre de problèmes récurrents, comme celui de l'origine du capitalisme. La société féodale était très bien régulée. Elle était fondée sur l'exploitation de la terre et des paysans. Sa destruction est due à des facteurs socioéconomiques. Mais surtout, elle renvoie au fait que la classe bourgeoise, la classe des marchands, a joué le rôle d'un virus corrupteur. Le passage au monde bourgeois ne s'explique pas par le fait que le moulin à vapeur a remplacé le moulin à vent. C'est un passage dû au fait que la société féodale n'avait plus le moyen de rétablir son homéostasie, sa propre régularité. Dans l'histoire humaine, il apparaît sans cesse des déviations. Si elles ne sont pas exterminées tout de suite - pensez au christianisme qui est une déviation du judaïsme, pensez à l'Islam qui est une déviation par rapport aux religions présentes à La Mecque à cette époque, pensez au socialisme et à la pensée de Marx - si ces déviations ne sont pas éliminées, disais-je, alors elles se développent, elles deviennent peu à peu des tendances, puis des forces sociales qui finissent par transformer la société (la religion, l'idéologie, etc.). Il faut rompre avec l'idée d'une histoire frontale qui avance majestueusement, tel un glacier, car l'histoire avance comme un crabe - elle avance de côté. C'est la même chose pour l'évolution biologique : un nouvel individu déviant apparaît et, s'il se consolide, il est à l'origine de la constitution d'une nouvelle espèce appelée à se développer.

Vous faites la proposition d'une science transdisciplinaire, qui penserait simultanément l'anthropologie, la sociologie et la politique pour appréhender la complexité du réel. Comment cette proposition se formule-t-elle théoriquement? Et, en pratique, comment pourrait-elle s'organiser, réunir des champs et des rythmes de travail qui sont différents?

Il faut bien voir une chose : on ne peut pas inventer une transdisciplinarité en partant des disciplines. On a besoin d'une pensée antérieure aux disciplines, d'une pensée qui soit complexe, transdisciplinaire par sa nature. Il s'est déjà produit dans l'histoire des pensées qui, par leur force, se sont montrées transdisciplinaires. Marx, par exemple, est un penseur capable d'aborder les problèmes philosophiques fondamentaux, de traiter les questions d'évolution, de sociologie, d'économie, de politique. Je pense aussi à Freud. Pour lui, le moi psychique se forme à partir d'une relation entre le ça - c'est-àdire, en fait, notre nature biologique, pulsionnelle, ce qui renvoie à la biologie - et le surmoi qui n'évoque pas seulement l'autorité du père ou du chef, mais aussi la société. La pensée de Freud touche donc à l'individu, à la société, à l'espèce humaine. Du reste, c'est pourquoi il effectue des incursions sur le problème des origines de l'humanité.

Comment m'apparaît le problème de l'anthropologie? D’abord, je prends ce mot " anthropologie" au sens qu'il avait au XIXe siècle, en Allemagne: connaissance de l'homme de nature réflexive, philosophique. Mais je pense aussi que cette connaissance doit se fonder sur les savoirs qu'apportent les sciences diverses que sont 
l'ethnographie, l'histoire, la sociologie... Elle peut également se nourrir de choses qui ne sont pas généralement reconnues comme sciences: je pense à la littérature et à la poésie. Dans la connaissance de l'être humain, la littérature - en particulier le roman occupe une place fondamentale. Mon point de départ, c'est que nous sommes enracinés dans le monde physique et biologique, et qu'en même temps, nous en sommes déracinés. J'insisterai ici sur la dimension de l'enracinement. Nous savons aujourd'hui que dans notre organisme, se trouvent des particules qui se sont formées, sans doute, aux origines de l'univers. Dans notre organisme, il y a aussi des atomes de carbone qui se sont formés dans un soleil antérieur au nôtre, il y a des molécules et des macromolécules qui se sont formées sur la planète Terre, il y a des cellules originaires qui se sont multipliées et différenciées. Nous savons que nous sommes des animaux, des vertébrés, des mammifères, des anthropoïdes. Nous savons aujourd'hui qu'il y a eu cette évolution que nous appelons l'hominisation, et qui, à travers le développement du cerveau, de la bipédie, conduit à l'émergence du langage humain, de la culture et de l'homo sapiens qui est l'étape ultime du «moi » au niveau biologique. Nous pouvons donc voir, aujourd'hui, comment nous sommes immergés dans l'univers naturel et comment nous en sortons.

Il suffit d'ailleurs que nous regardions en nous-mêmes pour voir que notre esprit (mind) est inséparable de notre cerveau, qui est un organe biologique. Il existe un lien très étroit entre notre psychique et notre soma. Non seulement notre esprit subit les maladies, les infirmités de notre corps, mais notre corps est modifié par notre esprit. Nous savons aussi que les actes les plus biologiques sont les plus culturels. Naître, qui est la chose la plus biologique, est aussi la chose la plus culturelle : il faut faire des déclarations à la mairie, des baptêmes, etc. Manger, qui est une chose biologique, est un acte rituel accompagné de tabous et de prescriptions alimentaires. Mourir, rien n'est plus biologique et rien n'est plus culturel. Et même, chier - comme on dit en termes polis, déféquer, faire des excréments - c'est une chose qui nécessite un papier spécial et des cabinets spéciaux. Cela veut dire que notre lien avec l'univers biologique et physique n'est pas seulement un lien chronologique, ni même seulement un lien permanent (c'est-à-dire, nous sommes toujours des animaux tout en étant des humains), c'est aussi un lien où les deux termes s'impliquent réciproquement.

Reste à opérer ce lien, à effectuer cette soudure, sur un plan épistémologique. Pour cela, on peut partir du concept d'auto-organisation. C'est un concept fondamental qui manque aussi bien en sociologie qu'en biologie. Bien entendu, quelques biologistes marginaux défendent ces idées d'auto-organisation. Pareil en sociologie (voir les travaux de Niklas Luhmann, en Allemagne). Mais tout cela reste marginal. La tendance dominante demeure liée à l'esprit de morcellement et de disjonction. Jusqu'à présent, les biologistes veulent tout expliquer par des molécules, des gênes et des programmes. Ils ne veulent pas concevoir l'organisation d'ensemble. Et la sociologie a une conception rigide et mécaniste du système social. L'idée d'auto-organisation, aussi bien pour les individus que pour la société, permet de faire la jonction entre l'univers biologique et le développement humain (avec ses phénomènes créatifs incontestables), sans tomber dans un réductionnisme.

Avec un tel schéma, on peut essayer de parvenir à une définition de l'humain. L'humain, c'est, d'un côté, des individus, bien évidemment. Mais d'un autre côté, ces individus font partie d'une espèce et d'une société. Ce ne sont pas seulement les individus qui sont dans la société. C'est aussi la société qui est dans l'individu - puisque, 
dans l'individu, dès la naissance, il y a le langage, la culture, les rites, les tabous, les prohibitions... De même, ce n'est pas seulement que nous sommes dans l'espèce, mais c'est aussi l'espèce qui est en nous : nous avons des organes génitaux qui assurent la reproduction de cette espèce, etc. En disposant de ce macroconcept de l'humain, si j'ose dire, on comprend alors que l'humain ne se réduit ni à l'individu, ni à la société, ni à l'espèce biologique.

\section{Quels sont quelques-uns des résultats de cette anthropologie?}

Prenez par exemple le problème de l'unitas multiplex, de l'unité du multiple. C'est un point, typiquement, de pensée complexe parce que, dans la «pensée normale », quand on voit l'unité, on ne voit pas la diversité. C'est la fameuse polémique entre Voltaire et Herder. Voltaire disait que tous les humains sont pareils. Même les Chinois, ils ont des ambitions, des amours, des jalousies. On est tous semblables. Et Herder, au contraire, disait que les cultures sont irréductibles les unes aux autres et que les êtres humains sont différents justement en fonction de leur culture. La discussion se poursuit encore aujourd'hui. Ceux qui voient l'unité humaine ne voient qu'une unité abstraite, et ceux qui voient les cultures concrètes sont incapables de voir l'unité humaine. Il est évident qu'il y a une unité, une identité humaine, une identité génétique, anatomique, physiologique, cérébrale et que les différences sont très faibles. C'est un point fondamental. Tous les hommes, quelle que soit leur race, ont les mêmes dispositions cérébrales. Les variations s'observent beaucoup plus entre les individus qu'entre les ethnies. Et surtout, il y a une unité affective. Même une jeune fille sourde, muette et aveugle de naissance est capable de sourire, de rire et de pleurer. Cela veut dire que sourire, rire et pleurs ne sont pas des phénomènes apportés par la culture. Ce qui est vrai, c'est que, en fonction des cultures, il y a des modalités tout à fait différentes. Dans certaines cultures, il est déshonorant pour un homme de pleurer. Dans d'autres, au contraire, par exemple à l'époque romantique, il est normal de verser des torrents de larmes à la première émotion. On sourit dans des circonstances tout à fait différentes. Dans la tradition chinoise, les Chinois souriaient au moment des enterrements. Autrement dit, il y a des phénomènes d'unité mais extrêmement diversifiés. Et je pense que la richesse humaine est dans cette capacité qu'a l'unité humaine de créer des formes culturelles extrêmement diverses et extrêmement riches. Ce qui possède, tout de suite, une implication éthique et politique, surtout aujourd'hui : il faut sauver l'unité humaine, mais il faut sauver aussi les diversités culturelles.

On en arrive à cette idée fondamentale de l'unité dialogique: unité de la diversité, diversité dans l'unité.

Prenez la question de la différence des sexes. Il y a les femmes, et il y a les hommes. Pourtant chaque sexe a, de manière récessive, c'est-à-dire refoulée, les caractères de l'autre sexe. Nous autres, hommes, nous avons des seins, même s'ils ne nous servent pas à grand-chose. Les femmes ont un clitoris qui est un organe masculin atrophié. Il y a une différence anatomique, une différence physiologique, mais tout cela peut se mélanger. Il faut reconnaître la dualité du masculin et du féminin et que chacun possède les deux. Jung disait que chez l'homme, il y a davantage d'animus et chez la femme, davantage d'anima. C'est pourquoi la femme cherchait son animus chez l'homme et l'homme son anima chez la femme. Michelet aussi disait, dans une phrase très belle : «J'ai les deux sexes de l'esprit. » C'est-à-dire qu'il était capable d'avoir la sensibilité dite féminine et en même temps la puissance d'organisation. Nous ne pouvons donc concevoir, sur ce plan-là, la relation ni sur le plan d'une séparation non communicante, 
ni sur le plan d'une identification. Par exemple, aujourd'hui, ce que l'on appelle l'unisexe, cela ne veut pas dire qu'il y a un sexe commun entre homme et femme, cela veut dire que, dans notre civilisation, un certain nombre de choses qui étaient réservées aux hommes et d'autres reléguées aux femmes sont devenues communes. C'est-à-dire qu'il y a des choses communes aux deux sexes.

Abstraitement, on définit l'espèce humaine comme homo sapiens, que l'on relie à homo faber, puisque l'homme, parce que technicien, est aussi rationnel. Au XVIIIe siècle, on a inventé l'idée que l'homme est homo economicus, c'est-à-dire pseudo rationnel, toujours à la poursuite de son propre intérêt et, sur cette base, on a construit une vision euphorique et abstraite de l'humanité. L'anthropologie que j'ai voulu développer définit l'homme comme homo sapiens et demens en même temps. La folie n'est pas un résidu, c'est une caractéristique de fond de l'humanité. Dès le début, il y a eu un déferlement horrible de ce que les Grecs appelaient l'hybris, la démesure. On affirme aujourd'hui que les hommes de Neanderthal, qui vivaient en Europe, tranquillement, il y a entre cent mille ans et soixante mille ans, ont disparu en dix mille ans au moment de l'arrivée d' homo sapiens, sous l'effet d'une maladie ou d'une épidémie. Or, on peut tout aussi bien faire l'hypothèse qu'ils ont été exterminés par l'homo sapiens qui jouissait peut-être, au début, d'une suprématie technique. Les aborigènes d'Australie ont bien été exterminés, une grande partie des Indiens d'Amérique du Nord aussi. Il y a en l'humanité quelque chose de terrible, de délirant, de fou. Et, entre la rationalité et le délire, il y a toute une zone incertaine qui renvoie à l'affectivité. Aujourd'hui, il est démontré, d'après les travaux de Damasio, de Jean-Marie Vincent, qu'il n'existe pas une rationalité froide et pure; la rationalité est toujours liée à l'affectivité (même le mathématicien est passionné par ses mathématiques). Quand on observe les espaces du cerveau, on voit que les centres émotifs sont mobilisés en permanence. L'affectivité est reliée tout aussi bien à la rationalité qu'à la folie (quand elle entre dans l'hybris).

Il faut penser ces deux dimensions de l'espèce humaine en même temps, ne pas dire que chez l'homme, il y a cinquante pour cent de rationnel et cinquante pour cent de folie. Il y a des interférences entre les deux.

Quelles sont les implications de la pensée complexe sur le problème de l'éthique de la pensée, de la responsabilité des intellectuels?

La coupure entre connaissance scientifique et problèmes éthiques renvoie à des raisons de fond. Quand la science moderne s'est constituée, au XVIIe siècle, elle était extrêmement faible et a éprouvé le besoin de s'autonomiser par rapport à la théologie, à la politique, à l'éthique. Elle s'est donc donné une éthique bien à elle, connaître pour connaître sans se soucier des conséquences. La science s'est développée uniquement dans un but cognitif, en l'absence de toute préoccupation éthique. Au cours du XXe siècle, elle a pu acquérir des pouvoirs absolument gigantesques : pouvoir de destruction de l'ensemble de l'humanité (arme thermonucléaire, armes chimiques très sophistiquées), pouvoir de manipuler l'avenir de notre destin biologique, grâce aux manipulations génétiques - pas seulement en vue d'éliminer des gènes nocifs à la santé des gens, mais éventuellement aussi pour créer un jour des Untermenschen ou des Übermenschen ${ }^{1}$.

Le problème, c'est qu'il est très difficile, de l'intérieur de ce qu'on appelle la science classique, d'opérer une jonction entre connaissance scientifique et conscience réflexive, éthique. D'abord, parce que, comme l'a bien vu Husserl dès les années 1930, la science, 
qui a su développer des moyens extraordinairement subtils pour connaître les objets, demeure néanmoins incapable de rien comprendre aux sujets, à tout ce qui est subjectif, c'est-à-dire demeure incapable de se connaître elle-même. Le scientifique connaît tout des galaxies les plus lointaines, mais il ne se connaît pas lui-même. La science classique est déterministe, elle rend inconcevable l'idée même d'un minimum de responsabilité. Si l'on veut qu'il y ait responsabilité, il faut un minimum d'autonomie et de subjectivité. La science reste donc fondamentalement aveugle devant le problème de la responsabilité.

Ensuite, l'existence de disciplines séparées rend impossible d'aborder les problèmes fondamentaux. Ce n'est pas le cas avec cette science nouvelle qu'est l'écologie, qui rassemble en elle des disciplines très diverses traitant du monde physique, biologique et même humain (dans la mesure où nous perturbons la nature). Avec l'écologie, il devient possible de confronter nos connaissances scientifiques - même s'il ne s'agit que d'hypothèses scientifiques : réchauffement climatique, dégradation irréversible de la biosphère... - et les problèmes de responsabilité que cela pose. La science doit se transformer pour devenir une science complexe. Il y a déjà des prémisses à cette transformation: l'écologie, les sciences de la Terre, les sciences de la préhistoire (qui traitent la question de l'hominisation)... La cosmologie, elle aussi, présente des aspects intéressants, car elle permet de nous situer, en tant qu'êtres humains, dans le cosmos, ce qui implique, sur le plan éthico-politique, de renoncer au rêve de conquête du monde formulé par Bacon, Descartes, Buffon ou encore Marx. Même le simple rêve de maîtriser la nature, comme s'il s'agissait d'un monde d'objets, est devenu ridicule, parce que suicidaire. On va de plus en plus assister à une résurgence des problèmes éthiques. La question écologique - celle de la dégradation de notre biosphère - a déjà amené le philosophe Hans Jonas à élargir le principe de responsabilité. C'est dans cette direction que nous devons réfléchir à opérer des liens entre science et éthique. Nous ne sommes encore qu'au début d'une telle réflexion et surtout, nous ne sommes encore qu'au début d'une politique qui prendrait vraiment en charge ces problèmes, elle qui est si peu préoccupée d'éthique.

Vous évoquez aussi la nécessité, en quelque sorte, d'une nouvelle religion ou d'un nouveau mythe, capable de s'opposer à l'évangile de la catastrophe et de l'effondrement...

Il y a trois types de religion. Vous avez les religions avec plusieurs dieux. Vous avez les religions du salut, c'est-à-dire celles qui promettent l'immortalité. Les plus connues sont le christianisme et l'islam. Et enfin vous avez eu des religions qui ne savaient pas qu'elles étaient des religions. On peut considérer que le communisme, à l'époque de l'Union soviétique, a constitué une religion de salut terrestre, dont les éléments se trouvaient déjà chez Marx, avec la promesse d'un monde réconcilié, sans exploitation de l'homme par l'homme. Cette promesse a fait beaucoup de martyrs et beaucoup de bourreaux, comme toute grande religion.

Je parle, quant à moi, d'un autre type possible de religion, qui ne dépendrait plus de l'idée de salut - sois mon frère, parce que nous serons sauvés - mais de celle de perdition - sois mon frère, parce que nous sommes perdus sur cette terre. On ignore pourquoi on est né, pourquoi on est là, pourquoi on va mourir, pourquoi on vit. À mon sens, la religion sert à établir une fraternité de façon à rendre cette Terre plus vivable et cette vie moins ignoble. Je me fonde sur l'idée de Schicksalsgemeinschaft, de communauté de destin - idée très belle d'Otto Bauer, qu'il avait utilisée pour définir les patries. Désormais, cette idée s'applique à l'humanité tout entière. Nous sommes tous dans une 
communauté de destin. Nous sommes tous confrontés aux mêmes problèmes vitaux et aux mêmes menaces mortelles. À cela, s'ajoute que malgré notre diversité, nous partageons une identité humaine fondamentale. Tout concourt donc à considérer que la Terre est notre patrie. Cette idée n'exclut d'ailleurs pas les patries qui existent déjà (France, Allemagne, Turquie...). Elle englobe aussi l'Europe [...].

En quoi la crise actuelle que vous évoquez s'apparente-t-elle à une véritable crise de civilisation? Comment appréciez-vous le schéma forgé par Adorno et Horkheimer d'une Dialektik der Aufklärung (Dialectique de la Raison) pour rendre compte de cette crise?

Je crois en effet que l'un des éléments de cette crise de civilisation renvoie au fait que, à partir de la rationalité qui est critique et liée à l'Aufklärung, s'est dégagée une raison dite instrumentale - soit l'utilisation de techniques à des fins propres à homo demens (la folie, le délire humain). Auschwitz est une très belle illustration de la rationalisation technique au service du génocide. Alors que le goulag soviétique, lui, n'était pas aussi rationnel (beaucoup trop de choses annexes ont joué : le gel, le froid...).

Second problème : la raison, sans s'en rendre compte, est devenue mythe, c'est-à-dire que, dans sa lutte contre les mythes religieux, elle est devenue providentialiste. Elle s'est trop tôt exaltée, à tel point que Robespierre a voulu, pendant la Révolution, dresser un culte à la déesse Raison. La tendance à diviniser la raison n'a rien de rationnel, car la raison, en même temps que construire des théories cohérentes et adéquates au monde, doit être critique et autocritique. L'aspect le plus visible de cette crise concerne la science, parce que celle-ci est la force empirico-rationnelle la plus grande de l'humanité. Son développement fait qu'aujourd'hui elle est capable de se mettre au service du profit, ou bien encore des États, qui sont eux-mêmes des entités paranoïaques. Tous les éléments positifs de cette civilisation née en Occident - mais qui, depuis, s'est répandue sur l'ensemble de la planète - sont en crise. Le développement de l'individualisme, conçu comme développement de l'autonomie individuelle, qui est une chose très souhaitable, a entraîné des effets négatifs, en particulier la destruction des liens traditionnels de solidarité (la famille, le village, le quartier). Destruction de toute solidarité concrète. Cela a conduit au développement de grandes solitudes, non seulement psychiques, mais aussi matérielles : beaucoup de gens âgés sont seuls, abandonnés. Il n'y a pas eu un développement harmonieux de l'individualisme et des communautés. Le sociologue français Maffesoli évoque l'émergence d'un néo-tribalisme : on voit aujourd'hui, en particulier dans la jeunesse, la constitution de bandes, de groupes qui se réunissent, etc. Ce sont des communautés provisoires et fragiles. Dans la société civile, il y a donc une résistance à l'atomisation. La ville a été quelque chose de très libérateur dans le développement de notre civilisation par rapport au village, où tout le monde se surveillait. La ville était chantée comme un espace de liberté. Mais la ville est aussi un monde de stress, de misère, de pollution, etc. Il y a une crise de la mégapole. L'économie est ambiguë parce que le marché était un moyen extraordinaire du développement de tous les autres aspects de la civilisation. Tous les efforts de l'Europe ont été de réguler le marché, maintenant tout cela éclate. Là aussi, nous avons un problème avec l'économie dite néo-libérale aujourd'hui. Nous avons une grave crise parce qu'il n'existe plus la pseudo-solution représentée par l'Union soviétique ou la Chine de Mao. On s'est rendu compte qu'il ne s'agissait pas de bons modèles. Depuis 1930, des esprits recherchent une «troisième voie ». Cela a toujours échoué. Ce projet a été balayé par la Seconde Guerre mondiale. Aujourd'hui, ce qu'on appelle la « troisième voie » en Allemagne, en Angleterre, ce n'est 
qu'une plaisanterie, un vague slogan sans consistance. La grande promesse de cette civilisation, c'était le bonheur. Dès 1968, en Californie, la jeunesse, qui pourtant vit dans les meilleures conditions de confort possibles, s'aperçoit que le bien-être matériel n'est pas le bonheur. Dans nos grandes villes, la consommation de sédatifs, de calmants, d'euphorisants, de drogues de toutes sortes, indiquent un malaise profond. La promesse du bonheur ne s'est pas réalisée. Il y a plutôt eu une dégradation de la qualité de la vie.

Le terme de globalisation décrit l'un des aspects qui manifestent cette crise de civilisation. À l'occasion du FSM à Porto Alegre, vous distinguez deux logiques de mondialisation : le modèle dominant et un modèle alternatif.

La mondialisation a commencé au XVIe siècle [avec l'arrivée des Européens aux Amériques]. Elle a pris une forme terrible. Phénomène étonnant, l'Espagne a pu conquérir deux civilisations beaucoup plus riches qu'elle, mais qui ne possédaient pas les armes à feu. Ces civilisations se sont donc effondrées. Beaucoup de morts, d'esclavage, etc. Introduction de l'alcool, que le monde occidental avait intégré depuis des millénaires, mais que les Indiens ne connaissaient pas. Arrivée de certains microbes, comme la syphilis, en Europe. Il y a eu aussi des aspects positifs, comme l'introduction du blé et du cheval en Amérique, du maïs en Europe. On a assisté au début d'un processus de métissage, surtout en Amérique latine. Cette mondialisation a donc eu des effets terribles, et des aspects secondaires féconds.

En réaction aux aspects les plus destructeurs, il y a eu une seconde mondialisation. Bartholomé de Las Casas, le prêtre espagnol, défend l'idée que les Indiens d'Amérique sont des humains comme les autres, bien que le Christ ne se soit jamais promené jusqu'en Amérique latine. Il a quand même réussi à emporter l'adhésion de l'Église. Montaigne, à la même époque, dit que toutes les civilisations ont leur vertu, leur valeur, et parvient, à sa façon, à ne pas se laisser enfermer dans l'occidentalocentrisme. Plus tard, Montesquieu rédige les Lettres persanes. Autrement dit, il naît un contre-courant dans les pays dominateurs, où se développent les idées de l'habeas corpus, de droits de l'homme, de démocratie et de nation. Ces idées, à un moment donné, vont être utilisées par les pays dominés pour s'émanciper. Donc, il y a un double processus. Ces deux mondialisations sont à la fois antagonistes et inséparables. Malheureusement, la première est la plus forte et se manifeste surtout sur le plan économique. Mais la seconde se manifeste également, sur d'autres plans, avec la diffusion des idées de démocratie. Vous avez des gens qui se comportent comme des citoyens du monde dans des ONG comme Médecins sans Frontières, Greenpeace, Amnesty International. On n'en est plus aujourd'hui à la vision rigide d'une antimondialisation refermée sur les frontières, qui prédominait jusqu'aux événements de Seattle. Avec des personnalités comme José Bové (qui joue le rôle d'un catalyseur), avec des lieux comme Porto Alegre, cette seconde mondialisation est en train de prendre son vrai visage.

Pensez-vous que le capitalisme ait un avenir? Peut-il se moderniser ou le modèle de croissance qu'il représente va-t-il s'effondrer? Est-il toujours orienté vers la production de masse? Ou bien suit-il exclusivement le principe de plus-value?

L'organisation sous forme de multinationales permet de s'affranchir de pas mal de barrières de contrôle étatique. Et le capitalisme a fait entrer, dans son champ, des domaines qui semblaient absolument irréductibles, comme les gènes. Pensons à une entreprise comme Monsanto, qui produit des OGM. On n'aurait pas pu imaginer que les gènes, que la biologie puissent devenir des marchandises. Le phénomène de la marchandisation de toute chose s'est accru. On voit très bien que les formes de 
résistance ne sont possibles qu'à la condition d'être elles-mêmes multinationales et internationales. Regardez les résistances aux OGM : c'est une résistance qui est inégale selon les pays, néanmoins, même aux Etats-Unis, les syndicats d'agriculteurs et les groupes de consommateurs sont parfois contre les OGM. Il est évident que le jour où il $\mathrm{y}$ aura de vraies internationales citoyennes qui se créeront, elles constitueront des forces de pression dotées d'un pouvoir de boycott, qui pourront également s'appuyer sur le développement d'une nouvelle économie qui elle-même reste embryonnaire - ce qu'on appelle aujourd'hui l'économie solidaire, vouée à la satisfaction des besoins sociaux. Même dans un pays comme la France, nombre de métiers d'aide aux personnes en difficulté (malheureuses, infirmes, isolées, etc.) pourraient être créés. Il existe déjà un embryon d'économie solidaire, c'est-à-dire d'une économie plurielle, non pas fondée uniquement sur le profit capitaliste, mais fondée sur une pluralité. Et même à l'intérieur $\mathrm{du}$ monde capitaliste, un certain nombre de personnes s'interrogent sur ce qu'on appelle l'entreprise citoyenne, ne serait-ce que pour une question d'image vis-à-vis du reste de la société ; ils éprouvent le besoin de se montrer sous un jour bienfaisant. Ils ne peuvent plus se contenter de créer des fondations pour aider les gens défavorisés, ni d'investir dans l'art. Il se développe aujourd'hui un nouveau type de relation à la fois antagonique et de complémentarité entre le monde du capital et le monde social.

Le capitalisme a des limites théoriques encore inaperçues. Il suscite des antagonismes réels, encore insuffisamment développés et coordonnés. L'alternative n'est pas de supprimer le marché, mais de le contrôler et de le réguler. Mettre l'économie au service de l'homme et non pas l'homme au service de l'économie. Le sort du capitalisme en dépend. Le risque de toute critique, c'est de rester soit trop hétérogène, soit trop unifiée. La critique donne lieu à la formation de multiples sectes qui noyautent la chose et font tout échouer. C'est le cas, par exemple, en France, avec les mouvements étudiants. Il s'agit néanmoins de phénomènes importants qui peuvent servir de catalyseurs pour une coalition mondiale, par exemple sur les questions d'écologie. Un début de mobilisation a permis la mobilisation des États lors de conférences comme à Rio ou à Tokyo. Nous entrons dans une nouvelle ère politique, semblable à celle des débuts du socialisme.

Les États nationaux peuvent-ils servir de contrepoint à cette globalisation ? Ou bien l'avenir est-il à des confédérations comme l'Union européenne? À quel niveau se situer pour réduire le pouvoir des trusts, pour changer le rapport de force entre les multinationales et les institutions?

Les États nationaux sont confrontés à des problèmes qui échappent à leur pouvoir et qui se posent à l'échelle soit de l'Europe, soit de toute la planète. Il faut donc que se créent des instances fédérales et confédérales. C'est un mouvement analogue à celui qui a conduit à la suppression de la monarchie absolue et à son remplacement par une monarchie constitutionnelle. Il est temps de supprimer le pouvoir absolu des États visà-vis des États étrangers. Cela signifie aussi qu'il faut sauvegarder les États nationaux. Une démocratie, ne serait-ce qu'à l'échelle européenne, c'est déjà très difficile, car il n'existe pas encore de partis et de syndicats transnationaux. Quand on veut créer une institution internationale, par exemple l'ONU, on se confronte toujours au problème que celle-ci reste aux mains des grandes puissances (grâce, par exemple, à des mécanismes comme le droit de veto).

L'Europe doit s'unir. C'est le cas aussi des pays d'Amérique latine, qui possèdent presque une langue commune. Cela serait également très utile aux pays du Maghreb et 
à l'ensemble des pays arabes, qui ont en commun une langue et une culture. Ce serait tout aussi bien pour l'Afrique noire, qui reste morcelée en tribus, et dont les seules divisions sont issues des anciens empires coloniaux. L'avenir, à mon avis, est aux confédérations. Il faut créer des instances au niveau planétaire, comme le proposait Gorbatchev : un conseil économique mondial, un conseil culturel mondial, un conseil écologique mondial, dotés de réels pouvoirs de décisions. N'envisageons pas un gouvernement mondial ni un super Etat mondial. Mieux vaut une confédération de type anarchiste, laissant de grandes libertés, mais avec des instances de décision pour les questions de vie ou de mort.

Il y a dix ans, vous avez rédigé un livre sur l'Europe dans lequel vous vous montriez très optimiste sur les possibilités de développement et d'intégration à l'échelle européenne. Aujourd'hui, quel est votre constat? Nous avons tendance plutôt à penser que la logique d'intégration se heurte aux vieilles logiques de puissance nationale (France, Allemagne, Royaume-Uni). II y a aussi le problème de l'intégration culturelle, avec le retour des profondeurs d'une identité chrétienne qui empêche de penser le rapport de l'Europe à la Russie, à la Turquie, etc.

Les logiques nationalistes dans les différentes parties intégrantes de l'Europe, dans les grandes puissances comme dans les petites - qui craignent d'être abandonnées ou satellisées par les grandes - restent extrêmement fortes. L'entente franco-allemande, qui fut un des moteurs de l'Europe, est aujourd'hui un peu fissurée. L'idée européenne telle qu'elle était défendue par Monnet, Schuman et d'autres était une idée politique. Dans le contexte de la guerre froide, il s'agissait de dire : « Unissons-nous, car il y a une grande puissance qui s'appelle l'Union soviétique ». Mais cette idée a été stoppée. Dans les années 1950, il y a eu un essor économique formidable, qui aurait dû n'être qu'une étape pour aborder les problèmes culturels et économiques, mais on n'a pas dépassé ce stade économique. Aujourd'hui, avec l'effondrement du rideau de fer, des pays comme la Pologne, la République Tchèque, etc. sont membres de droit de cette Europe - ce qui oblige à trouver une solution sur la question de savoir si l'on conserve ou pas le vote à l'unanimité !

En ce qui concerne la Russie, le problème n'est pas qu'elle soit de tradition orthodoxe ; la Roumanie par exemple, pourrait fort bien être intégrée à l'Europe... Le problème est qu'il s'agit d'une masse immense, pour laquelle il faut trouver un statut d'association spécial. La Turquie est le premier pays d'origine islamique qui se soit laïcisé et donné des bases potentiellement démocratiques - malgré le fait qu'il s'agisse d'une dictature. Il est indispensable que des pays qui ne soient pas d'origine chrétienne intègrent l'Europe. D'abord, parce que plusieurs Islams européens subsistent de l'Empire Ottoman (une grande partie de l'Albanie, le Kosovo). Ensuite, parce qu'il existe trois ou quatre millions de musulmans en France, des musulmans d'origine turque en Allemagne, en Autriche, etc. Je suis donc, pour des raisons politiques et culturelles, tout à fait partisan de l'intégration de la Turquie dans l'Europe, étant donné qu'elle possède une base laïque. Mais il y a des résistances... L'Europe ne peut être l'Europe qu'à la condition qu'elle s'ouvre. L'Europe moderne s'est faite sur la laïcité, en refoulant les pouvoirs du catholicisme. L'Europe doit poursuivre cette tâche, c'est pourquoi j'accorde une valeur symbolique à la question de la Turquie. En plus de cela, il faut bien voir que tous les éléments démocratiques en Turquie aspirent à entrer dans l'Europe, alors que les éléments les plus régressifs et réactionnaires regardent plutôt vers l'Asie. 


\section{Comment penser le rapport entre les différentes cultures et les différentes religions?}

Prenez les grandes religions monothéistes. Il existe une énorme différence entre leur source, qui est universaliste, et leur manifestation, qui est particulière et dogmatique. La source du christianisme est universelle et évangélique, ce qui n'a pas empêché l'Eglise de faire les croisades. Les religions peuvent évoluer en retournant à leur source. Elles possèdent un caractère progressiste. Une culture est toujours à la fois ouverte et fermée, fermée afin de sauvegarder son intégrité, son identité, ouverte parce qu'elle intègre des éléments venus d'autres civilisations. Au Brésil, par exemple, il y a des phénomènes de métissage, des phénomènes culturels créateurs: il y a une culture métissée, produit du mélange entre les Noirs, les Portugais... Regardez le flamenco, qui en lui-même est déjà le produit d'un syncrétisme (car les Gitans viennent d'Inde et il subsiste dans cette musique des éléments indiens, comme le battement des mains) : il y a aujourd'hui le flamenco rock, etc. Même chose pour le raï en Afrique du Nord. Une culture trop faible, en s'ouvrant, se désintègre : elle meurt, elle est condamnée, car elle n'a pas la force de vivre par elle-même. Dans l'histoire, on a détruit des cultures qui ne demandaient qu'à vivre. Toujours au Brésil, beaucoup de petites cultures indigènes ont été massacrées. Ces "petites cultures " sont menacées par des cultures très fortes et dominantes. C'est un problème délicat, car si on les intègre, elles se désintègrent aussi. Mais souvent, ces cultures résistent (c'est le cas de la culture bretonne, corse, etc.). Il ne faut pas craindre le métissage.

Qu'entendez-vous par anthropolitique, ce que vous appelez aussi une politique de civilisation?

L'idée d'anthropolitique, c'est l'idée d'une politique de l'homme, au sens générique. Je suis parti du constat que les bases de la pensée de Marx étaient insuffisantes, qu'il fallait aussi puiser ailleurs à d'autres sources scientifiques. L'idée d'anthropolitique, c'est aussi l'idée qu'on peut améliorer l'être humain - je pense à la thématique de l'amour qu'on trouve dans la religion, dans le surréalisme aussi. La politique s'est gonflée de tous les problèmes humains. Il ne faut pas réduire ces problèmes à la politique, il ne faut pas plus dissoudre la politique dans les problèmes humains. La politique doit néanmoins prendre en charge beaucoup de choses : elle doit devenir, non pas totalitaire, mais multidimensionnelle. L'idée d'une politique de la civilisation m'est venue, car notre civilisation actuelle pose désormais autant de problèmes qu'elle ne présente de solutions et d'avantages. Les modèles de développement, qu'on a appliqués au tiers-monde, étaient fondés sur l'idée fausse que ce sont la technique et l'économie qui vont apporter le développement humain. Le problème de notre civilisation occidentale est qu'elle a créé un sous-développement humain et moral, en raison du développement de l'égocentrisme et de l'égoïsme. On a pu assister ensuite à une sorte de revanche du qualitatif sur le quantitatif [...]. La qualité de la vie correspond à une aspiration fondamentale. Ce qui n'empêche pas, bien sûr, que le problème de la quantité se pose dans certains pays, qui n'ont pas suffisamment de ressources...

Prenez une ville comme Paris. Elle souffre de phénomènes pathologiques caractérisés : fatigue, stress... Les dépenses de soins pour tous les maux qui résultent de cette vie urbaine sont considérables. Il faut créer, par exemple, des parkings souterrains, de façon à ce que la ville soit livrée à des transports publics non polluants : tramway, zone piétonne, etc. Il faut régénérer la vie. En plus, cela donnerait du travail, et du travail qui a du sens. Il existe des solutions pour lutter contre la désertification des campagnes : grâce aux ordinateurs et au télétravail, on peut vivre en connexion totale tout en étant 
isolé. La campagne est soit désertée, soit livrée aux grandes exploitations, qui ellesmêmes sont fondées sur les principes quantitatifs de rendement, et non sur la qualité. Aider le développement de l'agriculture biologique, aider à la résurrection des moyennes entreprises, d'abord pour tout ce qui existe déjà (dans le vin...). Régénérer les campagnes, les villes, faire une politique de développement de la solidarité, créer des maisons de la solidarité dans tous les quartiers, renforcer et coordonner les choses positives qui existent déjà... Cela permettrait une vraie résurrection de l'avenir, du Zukunft. Non pas réduire la politique à l'écologie, non pas dissoudre la politique dans l'écologie, mais faire de l'écologie une dimension de toute politique locale, nationale, européenne et mondiale. Le problème des relations avec la biosphère est devenu un problème politique. C'était ça, mon idée de politique de civilisation - une politique fondée sur des valeurs. Je pense que la politique n'a jamais cessé d'être en relation avec certaines valeurs; je pense même que dans l'idée de souveraineté, il y a l'idée du bon souverain. Le fait que la politique se voue à la collectivité et à la cité signifie que la politique doit faire le moins de mal possible à la cité. L'entrée active en politique de ce qu'on peut appeler le peuple, ou les peuples, aboutit à la devise: liberté, égalité, fraternité. Notons que le mot de fraternité n'apparait qu'en 1848. À l'époque de la révolution, la devise était seulement : liberté et égalité. La référence à la fraternité est un peu le produit de l'influence du socialisme. Ces trois termes sont complémentaires, mais aussi antagonistes, car la liberté toute seule détruit l'égalité et la fraternité (c'est le problème aujourd'hui avec l'émergence d'un marché mondial, qui montre bien que la liberté économique est destructrice). De même, l'égalité, quand elle est imposée, tue la liberté. Quant à la fraternité, le problème est qu'elle ne se réduit pas à une position politique: c'est un sentiment vécu, de Gemeinschaft (communauté). Cette devise «liberté, égalité, fraternité » est donc extrêmement complexe. La relation entre éthique et politique est à la fois complémentaire et antagonique. Ce qu'on appelle la Realpolitik ne peut pas être balayé d'un revers de main, sous prétexte qu'elle serait cynique. Je ne crois pas que la politique puisse se soumettre entièrement à l'éthique.

Autre difficulté, le conflit de l'utopisme et du réalisme. Je préfère l'utopisme au réalisme. Néanmoins, une critique conjointe de l'utopie et du réalisme est nécessaire. Il existe deux genres d'utopie. L'utopie d'une société parfaite, ordonnée, harmonieuse, où tous les problèmes humains seraient résolus. C'est une mauvaise utopie car, pour l'imposer, il faut brutaliser la réalité humaine. La bonne utopie est de penser qu'aujourd'hui certaines choses, que l'on croit impossibles, sont possibles. Des guerres se déchaînent un peu partout, pourtant il est possible de parvenir à une paix. Dans un pays comme la France, il y a eu des guerres entre seigneurs féodaux, qui ont pu être supprimées grâce à l'instauration d'une autorité supérieure. La " mauvaise » utopie, c'est le meilleur des mondes, la "bonne» utopie, c'est un monde meilleur. Autre difficulté : il existe une «écologie de l'action », c'est-à-dire qu'une action échappe à ses intentions, obéit à une dialectique du milieu politique et social dans lequel elle s'inscrit et parfois, dans un sens contraire à celui désiré. La tentative de révolution en Espagne aboutit à l'instauration du régime de Franco. La tentative de réaction aristocratique en France en 1788 provoque la Révolution française. Chirac dissout l'Assemblée nationale pour consolider sa majorité et offre le pouvoir à Jospin. Il faut une stratégie pour être sûr que les intentions de son action ne soient pas détournées. Il n'y a pas de recette, Saint-Just a eu cette phrase admirable : « Tous les arts ont produit leurs merveilles, seul l'art de gouverner n'a produit que des monstres. » 
Dernière question : les aspects pratiques de la politique hexagonale...

Dans le cadre de la gauche plurielle, les socialistes n'ont fait aucun investissement politique, pris dans une stratégie de pouvoir, de conservation de pouvoir. Pareil pour les Verts. Même sur le plan écologique, leur bilan est très faible. Aucune formation politique actuelle ne peut assumer cette politique de civilisation. Peut-être faut-il penser que seules de vastes ligues, de vastes convergences, en sont capables. Il y a des mouvements comme ATTAC, par exemple, sur le plan économique. La force et la forme de cette politique ne sont pas encore cristallisées. Je pense que, comme toujours, il va falloir une poussée très forte. L'écologie n'a été vraiment prise en compte que suite à des catastrophes (par exemple celle de Tchernobyl), à des alertes mythico-réelles comme la diminution de la couche d'ozone sur les pôles, à la prise de conscience que toute la pollution de la société moderne va provoquer un réchauffement de la planète, avec les conséquences catastrophiques que l'on sait. Je pense que la prise de conscience des problèmes qualitatifs va nécessairement advenir. Mais il est impossible de déterminer, lorsqu'un mouvement est naissant, à quel moment les idées qu'il porte vont devenir une force organisatrice et organisée. Prenez la naissance du christianisme. Entre la prédiction de Paul et la structuration de l'Eglise, il a fallu attendre deux siècles. De même, une trentaine d'années ont été nécessaires pour que se crée le premier parti social-démocrate, en Allemagne. Aujourd'hui, on peut seulement tout faire pour que se développe une déviance, pour que la prise de conscience donne lieu à la naissance d'une force organisée. Mais l'heure n'est pas à lui donner tout de suite un modèle, ce qui serait artificiel. Il faut établir des convergences, et puis à un moment donné, cela va produire un changement - du quantitatif au qualitatif, comme disait Engels. Il se produit un changement qualitatif lorsque, à partir d'une certaine quantité, cela donne lieu à une morphologie nouvelle. Aujourd'hui, un certain nombre de volontés sont en train de faire se cristalliser la forme nouvelle qui va naître. Nul besoin d'être programmatique. Déjà des mesures sont prises (par exemple celles qui favorisent l'agriculture biologique). Beaucoup de petites mesures vont dans ce sens-là, mais elles ne sont pas encore agrégées...

\section{NOTES}

1. « Sous-hommes » et « surhommes », termes utilisés par la propagande nazie. NDR. 